\title{
MINIATURIZATION OF SINGLE LAYER U-SLOT MICROSTRIP PATCH ANTENNA
}

\author{
Jisha M. V', Abisha T. P ${ }^{2}$, Neelima K. $\mathbf{P}^{3}$, Raisha Rahim ${ }^{4}$, Shalu $\mathbf{N}^{5}$, Syamly S. B \\ ${ }^{I}$ Electronics and Communication Engineering, Cochin University of Science And Technology, College of Engineering \\ Vadakara \\ ${ }^{2}$ Electronics and Communication Engineering, Cochin University of Science And Technology, College of Engineering \\ Vadakara \\ ${ }^{3}$ Electronics and Communication Engineering, Cochin University of Science And Technology, College of Engineering \\ Vadakara \\ ${ }^{4}$ Electronics and Communication Engineering, Cochin University of Science And Technology, College of Engineering \\ Vadakara \\ ${ }^{5}$ Electronics and Communication Engineering, Cochin University of Science And Technology, College of Engineering \\ Vadakara \\ ${ }^{6}$ Electronics and Communication Engineering, Cochin University of Science And Technology, College of Engineering \\ Vadakara
}

\begin{abstract}
A microstrip patch antenna, in its simplest form consists of a radiating patch on one side of a dielectric substrate and a ground plane on the other side. Typically a patch antenna has low profile, low cost and reduced weight. Here Ushaped antenna is implemented on FR4 substrate. Rectangular shaped ground plane is used. The simulated result is verified experimentally. The proposed antenna is simple in structure. It is highly preferable in wireless communication. This antenna is designed for $3.6 \mathrm{GHz}$ frequency which lies in UWB range. The antenna size has been reduced to $25 X 20 \mathrm{~mm}$.
\end{abstract}

Keywords-MSA, UWB, FEM, HFSS, Microstrip Antennas, Slot Antennas ****

\section{INTRODUCTION}

APatch antenna is a type of radio antenna with a low profile, which can be mounted on a flat surface. It consists of a flat rectangular sheet or 'patch' of metal, mounted over a larger sheet of metal called a ground plane. A substrate is placed between the two metal plates. Patch and ground are conducting plane, while substrate is a non conducting plane. Microstrip antennas are low profile applications at frequencies above $100 \mathrm{MHz}\left(\lambda_{0}<3 \mathrm{~m}\right)$. Radiation from the microstrip antennas can occur from the fringing fields between the periphery of the patch and the ground plane. The length $\mathrm{L}$ of the rectangular patch for the fundamental TM10 mode excitation is slightly smaller than $\lambda / 2$, where $\lambda$ is the wavelength in the dielectric medium, is which in terms of free- space wavelength $\lambda_{0}$ is given as $\lambda_{0} / \sqrt{\epsilon_{c}}$, where _e is the effective dielectric dielectric constant of a microstrip line of width $\mathrm{W}$.

$\lambda$

Return loss is an important parameter to calculate the input and out put power, because if the load is mismatched the whole power is not delivered to load. If the return loss is very low, then the patch is radiating well. In this paper the return loss has reduced to a great extend.

\section{ANTENNA DESIGN}

Antenna is designed based on the equations [1],[3] shown below,

The calculation of $\epsilon_{\text {reff } f}$ is given by the equation

$$
\epsilon_{\text {reff }}=\frac{\epsilon_{r}+1}{2}+\frac{\epsilon_{r}-1}{2}\left[1+12 \frac{h}{W}\right]^{-\frac{1}{2}}
$$

The length extension is given as:

Substituing the value of $\epsilon_{\text {reff } f}, W$ and $h$,

$$
\Delta L=0.412 h \frac{\left(\epsilon_{\text {reff }}-0.3\right)\left(\frac{W}{h}+0.264\right)}{\left(\epsilon_{\text {reff }}-0.258\right)\left(\frac{W}{h}+0.8\right)}
$$

Substituing the value of $\epsilon_{\text {reff }}$ and $f_{r}$

$$
L_{e f f}=\frac{c_{0}}{2 f_{r} \sqrt{\epsilon_{r e f f}}-2 \Delta W}
$$

The actual length of the antenna is given by the equation:

$$
L=L_{e f f}-2 \Delta L
$$

The transmission model is applicable to infinite ground planes only. However for practical considerations, it is essential to have a finite and infinite ground plane can be obtained if the size of the ground plane is greater than the patch dimensions by approximately six times the substrate thickness all around the periphery. 
Ground plane dimension is given by:

$$
\begin{aligned}
& W_{g}=2 \times 6 h+w \\
& L_{g}=2 \times 6 h+L
\end{aligned}
$$

According to the design equations antenna dimensions are obtained. For $3.6 \mathrm{GHz}$ frequency the dimensions are large. By proper optimization, the size got reduced. Finally we reached a better result of dimension and is shown Fig.1

\section{ANTENNA STRUCTURE}

The antenna as shown in the Fig.1 has a U-slotted patch separated from the rectangular ground plane by a FR4 substrate of $\epsilon_{\mathrm{r}}=4: 4$ and $\mathrm{h}=1: 6 \mathrm{~mm}$. We have designed number of U-slotted antennas having different dimensions. As per the reference paper [1] the antenna dimension is too large and our intention was to reduce the size to a great extend. Finally the size got optimized and reached in a dimension of $25 \times 20 \mathrm{~mm}$ which can even be used in mobile phones. The patch dimension is only $15 \times 10 \mathrm{~mm}$ and the slot is $10 \times 7 \mathrm{~mm}$.

$\epsilon$

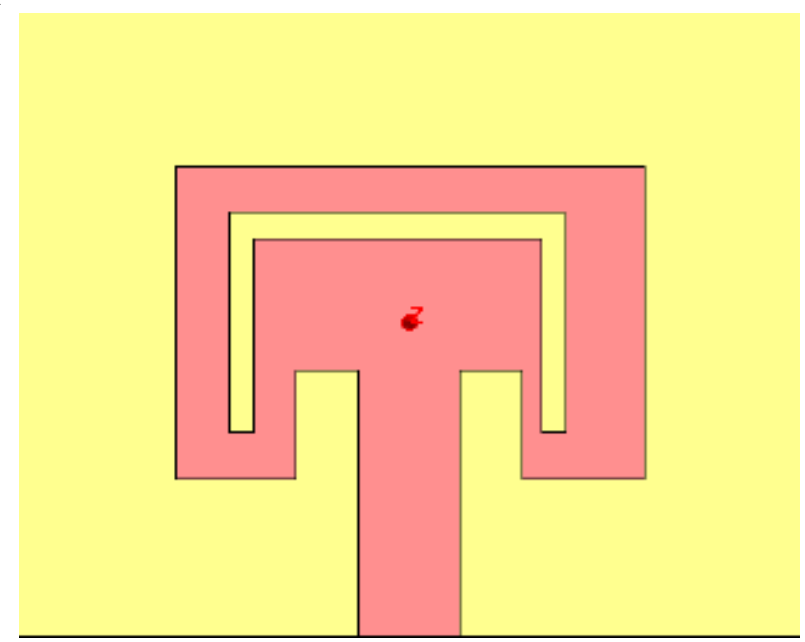

Fig. 1. Structure of U-slot antenna

Two designs finalized. These designs having the difference only in the position of the slot. In one case the slot is kept at the middle of the patch and in the other slot moved sidewise. The former structure gave high return loss (between -6 to $15 \mathrm{~dB}$ ) while latter gave better result and the obtained return loss is $-26.9154 \mathrm{~dB}$. Another important feature employed in this structure is inset feed.

\section{RESULT ANALYSIS}

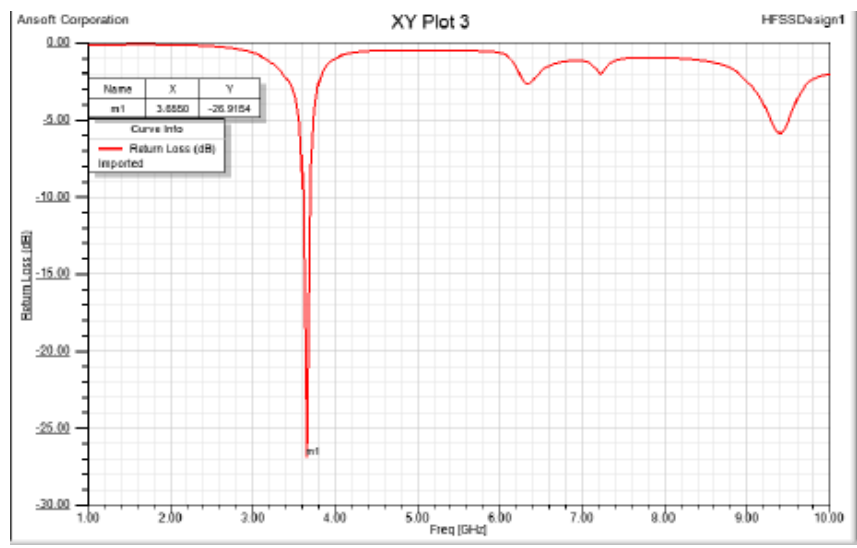

Fig. 2. Return Loss: Simulated result

This antenna is designed for miniaturization, getting better return loss and to operate in broad band application. Different steps has been utilized to get a better result. By using HFSS software analysis has done. The improvement seen only in the size reduction and high return loss as shown in the Fig.2. Fabrication result also shows the same (Fig.3). The band width has not improved much. By using a substrate of high thickness or using substrates having low dielectric constants bandwidth can be improved. Only a small variation is observed in the measured result from the simulated one.

\section{CONCLUSION}

In this paper so far discussing about a typical U-slot patch antenna. From the base paper [1] lots of improvement have been done. The antenna described in the base paper had lots of disadvantages. They are: antenna is larger in size , reduced return loss, reduced bandwidth, coaxial probe feeding were used and hence were difficult to design. MoM (Method of Moment) were employed in the base paper.

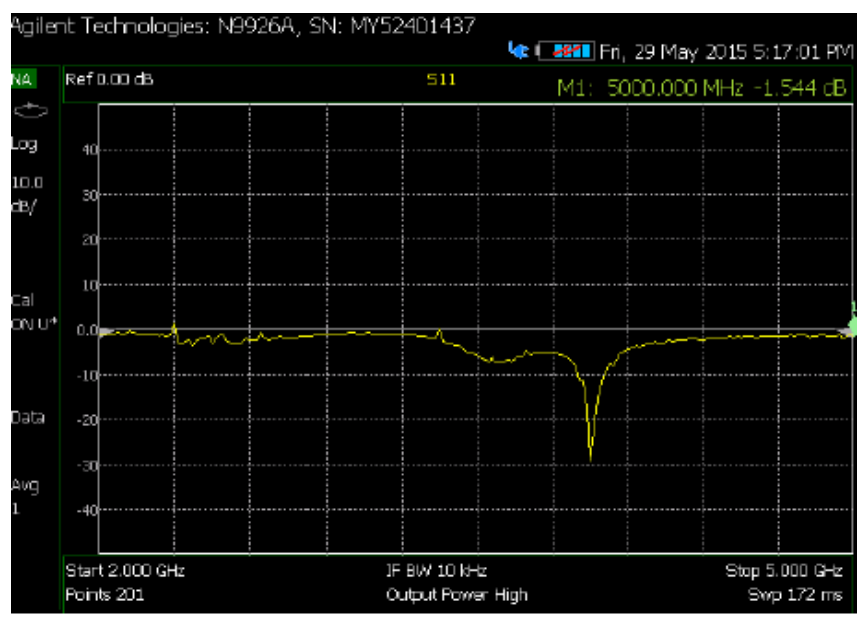

Fig. 3. Return loss: Measured result 
In this paper FEM method in frequency domain is employed. By proper design, the antenna size got reduced, obtained better return loss (approximately -25dB). Since we used microstrip inset feeding technique, radiation loss problems were avoided that caused due to coaxial feeding.

Even we achieved better performance by using this antenna, there is still some difficulties existing, such as required bandwidth is not obtained.

This can be improved by using substrate of high relative permittivity, reduced substrate thickness, making double layer substrate etc.

\section{REFERENCES}

[1]. Steven Weigand, Member, IEEE, Greg H. Huff, Kankan H. Pan, and Jennifer T. Bernhard, Senior Member, IEEE, "Analysis and Design of Broad-Band Single-Layer Rectangular U-Slot Microstrip Patch Antennas," IEEE transactions on antennas and propagation, vol. 51, no. 3, March 2003.

[2]. Kin-Fai Tong, Kwai-Man Luk, Senior Member, IEEE, Kai-Fong Lee, Fellow, IEEE, and Richard Q. Lee, "A Broad-Band U-Slot Rectangular Patch Antenna on a Microwave Substrate", IEEE transactions on antennas and propagation, vol. 48, no. 6, June 2000.

[3]. C.A. Balanis. "Antenna Theory: Analysis and Design", 2nd Ed, United States of America, John Wiley Sons, 0471592684, 1982.ch. 14 .

[4]. K.D. Prasad. "Antenna and Propagation".

[5]. J.D.Kraus, R.J Marhefka and Ahmed S Khan ,'Antennas for all applications', Tata Mc Graw Hill, 3rd edition.

[6]. American Journal of Engineering Research (AJER), 2013, Atser A. Roy, Joseph M. Mm, Gabriel A. Igwue Department of Electrical and Electronics Engineering, University of Agriculture, Makurdi, Nigeria, "Enhancing the Bandwidth of a Microstrip Patch Antenna using Slots Shaped Patch", .

[7]. Wei-Mei Li, Bo Liu, and Hong-Wei Zhao, "The UShaped Structure in Dual-Band Circularly Polarized Slot Antenna Design", IEEE Antennas and Wireless Propagation Letters, VOL. 13, 2014447. 